\title{
HERA Structure Functions
}

\section{Claude Vallée ${ }^{1}$}

Centre de Physique des Particules de Marseille

163 Avenue de Luminy

Case 902

13288 Marseille cedex 09

France

E-mail:vallee@cppm.in2p3.fr

On behalf of the ZEUS and H1 Collaborations

The H1 and ZEUS experiments have now completed their data collection and are in the process of extracting their final high precision results on the proton structure and its internal dynamics. The recent combination of the available published results from HERA I has significantly improved the knowledge of low x quark and gluon densities, which results in higher precision LHC predictions at the electro-weak scale. The determination of high $\mathrm{x}$ parton densities is still limited by statistical precision and will benefit from the analysis and combination of the full HERA II data samples. Investigations of the QCD dynamics through inclusive NLO QCD fits, as well as with measurements of the longitudinal structure function $\mathrm{F}_{\mathrm{L}}$ and of heavy quarks production, show that the DGLAP approximation is valid in the bulk of the phase space covered at HERA.

2008 Physics at LHC

Split, Croatia

September 29 - 4 October 2008

\footnotetext{
$1 \quad$ Speaker
} 


\section{Introduction}

The measurement of the proton structure at HERA is of crucial interest for LHC physics: electro-weak processes in proton-proton interactions at $\sqrt{S}_{\mathrm{S}}=14 \mathrm{TeV}$ involve initial partons with a proton momentum fraction $\mathrm{x}$ in a range $\sim 10^{-3}$ to $\sim 10^{-4}$, which is out of reach of fixed target experiments and can only be accessed at HERA. For the accuracy of LHC predictions, it is important not only to extract the parton distributions, but also to probe their QCD evolution over a large range, in order to safely extrapolate the measurements from 4-momentum transfers squared $\mathrm{Q}^{2}$ of a few $10 \mathrm{GeV}^{2}$, the typical scales at which they are measured at low $\mathrm{x}$ at HERA, to the $\mathrm{M}_{\mathrm{W}}{ }^{2}$ and higher scales at which they are needed at LHC.

This program can now be performed with high precision by the H1 and ZEUS experiments, using their final data samples collected from 1992 to 2007, which correspond to integrated luminosities of $\sim 0.5 \mathrm{fb}^{-1}$ per experiment. The data are approximately equally shared between electron- and positron-proton collisions. The last three months of data taking in 2007 were devoted to lower energy collisions for the measurement of the longitudinal structure function $\mathrm{F}_{\mathrm{L}}$.

The Neutral Current (NC) cross section is mediated by photon $(\gamma)$ and $Z^{0}$ exchange. After correction for the dominant $\gamma$ propagator effect, it is sensitive to three generalized structure functions $\mathrm{xF}_{3}, \mathrm{~F}_{2}$ and $\mathrm{F}_{\mathrm{L}}$, related to each other by helicity factors: $\mathrm{xF}_{3}$ directly measures the valence quark content at high $\mathrm{x}$ of the proton, $\mathrm{F}_{2}$ measures the sum of the sea and valence quark distributions, and $F_{L}$ is directly sensitive to the gluon content since spin $1 / 2$ quarks must have a finite transverse momentum (and hence, within QCD, arise from gluon splitting) to couple to longitudinal photons. The Charged Current (CC) cross-section, mediated by $\mathrm{W}^{ \pm}$exchange, allows the $\mathrm{u}$ and $\mathrm{d}$ valence distributions to be disentangled which dominate in electron- and positron-proton interactions, respectively.

\section{Parton Distributions}

High $\mathrm{x}$ valence distributions are best probed with high $\mathrm{Q}^{2} \mathrm{NC}$ and $\mathrm{CC}$ cross-sections. A first combined determination of $\mathrm{xF}_{3}$, based on data collected up to 2005, has been released by H1 and ZEUS [1]. The published HERA I CC cross sections have also been combined [2] and are displayed in figure 1 . The data show the expected factor two difference between the u-quark and d-quark densities, as probed by the electron-proton and positron-proton CC's, respectively. The precision of the data is still statistically limited and will benefit from the analysis and combination of the full final HERA II data samples.

The reduced $\mathrm{NC}$ cross section, approximately equal to the structure function $\mathrm{F}_{2}$, is shown in figure 2 as obtained [2] by combining the $\mathrm{H} 1$ and ZEUS published HERA I NC results. $\mathrm{F}_{2}$ is measured in a very large range in $\mathrm{x}$ and $\mathrm{Q}^{2}$. The H1-ZEUS combination not only improves the statistical errors, but also reduces the systematic uncertainties, thanks to the cross-calibration of both experiments in the combination procedure. The measurement reaches an unprecedented precision especially in the low $\mathrm{x}$ domain of relevance for LHC electro-weak processes. 


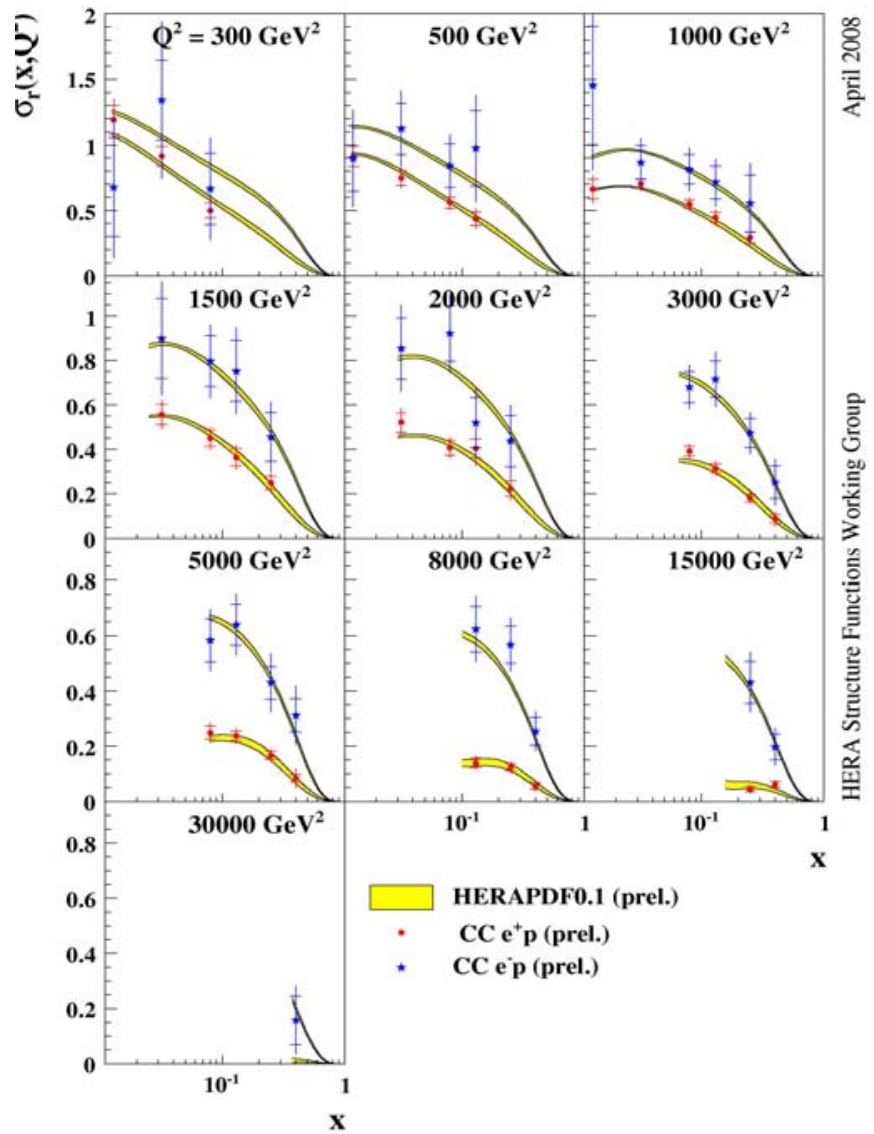

Figure 1: The reduced CC combined cross section from H1 and ZEUS published HERA I data.

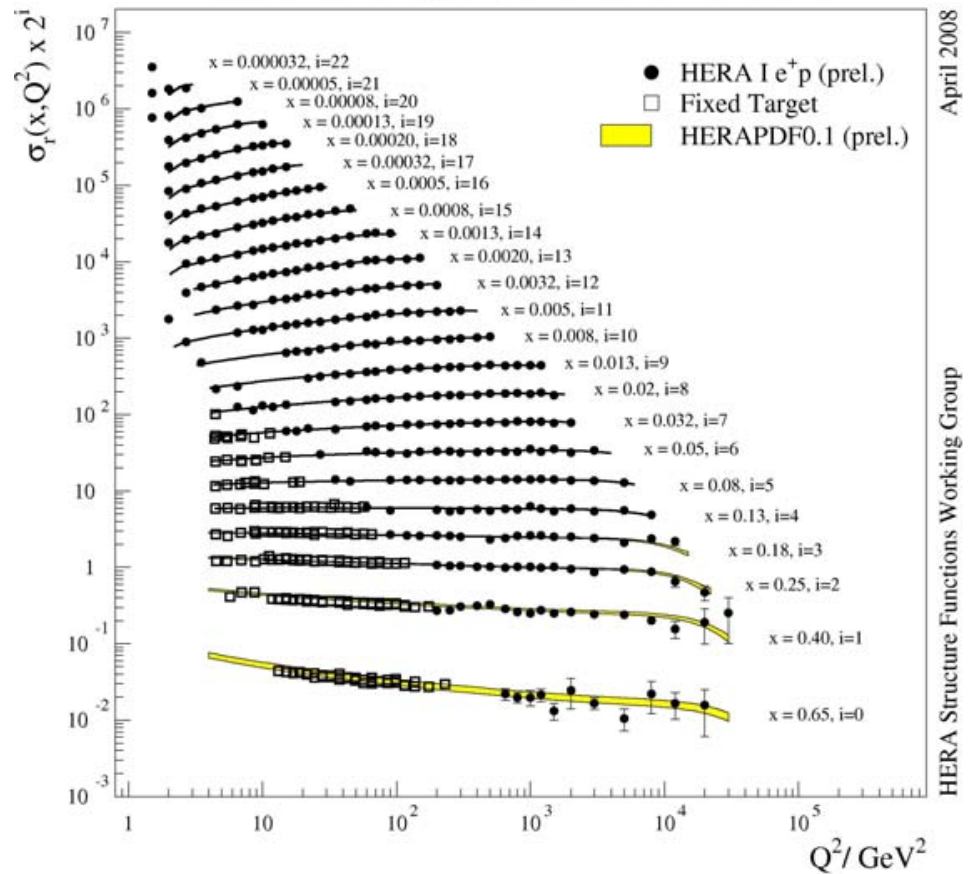

Figure 2: The reduced NC combined cross section from H1 and ZEUS published HERA I data. 
In order to extract the Parton Distribution Functions (PDFs) of the proton, the combined HERA I NC and CC cross sections are used as input of a common NLO QCD fit, named HERAPDF0.1. The fit procedure is optimized from the earlier individual PDF fits of each experiment. As shown in figure 2, NLO QCD in the DGLAP approximation remarkably describes the cross section scaling violations over the full range covered by the measurements. In figure 3 the individual HERAPDF0.1 parton distributions are shown in comparison with the previous individual fits of each experiment, based on the same data but prior to any combination. The improvement in precision brought by the combined data is impressive, especially for the low $\mathrm{x}$ gluon and sea quark distributions. It was checked that this improvement was indeed due to the improved precision of the input data, and not to a-priori over-constraints of the parton distribution shapes. In addition to experimental errors, the quoted PDF errors also include various model uncertainties associated to the assumptions made in the QCD fit. Due to the much improved experimental precision of the input data, model uncertainties now play an important role and will have to be considered with care in the future.
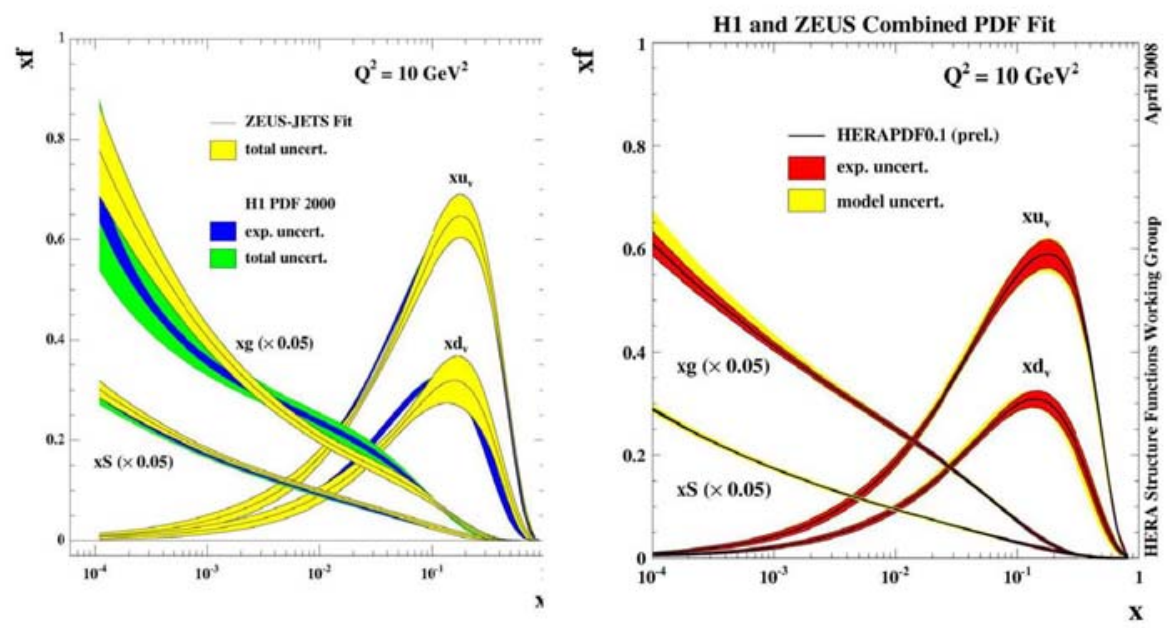

Figure 3: Parton distributions from the combined HERAPDF0.1 fit (right) compared to previous individual fits (left)
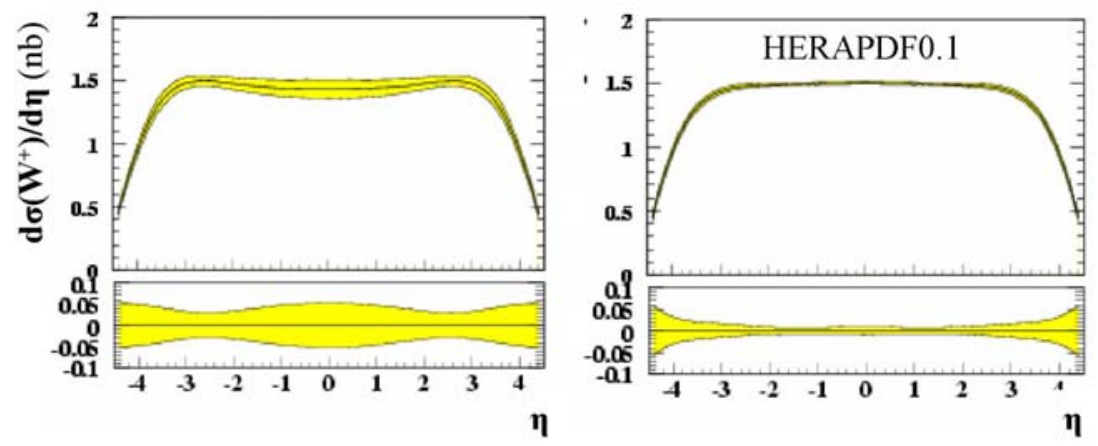

Figure 4: $W^{+}$cross section at LHC and associated PDF uncertainty as a function of rapidity, as predicted from the combined HERAPDF0.1 fit (right) compared to previous individual fits (left). 
The impact of the improved PDFs on LHC predictions is illustrated by figure 4 for W production [4]. The uncertainty of the predicted $\mathrm{W}$ cross section in the central region is reduced to 2-3\% with HERAPDF0.1. Preliminary studies [5] also show that the HERAPDF0.1 behave reasonably well for the gluon at high $\mathrm{x}$, though no Tevatron jet data are used in the fit. HERAPDF0.1 is now publicly available to LHC experiments within LHAPDF5.6.0 [6].

Considering the breakthrough in precision brought by the combined HERA I data, together with the prospects of the final HERA II data, a review of the optimal experimental inputs to global PDF fits and the estimation of model uncertainties are essential issues to be addressed by the PDF4LHC workshop in the near future, in order to produce the best input PDF's for LHC experiments.

\section{Tests of QCD Dynamics}

The two main ingredients of QCD dynamics are the strong coupling $\alpha_{\mathrm{s}}$ and the gluon content of the proton, which is generated dynamically through QCD radiation from quarks.

At HERA $\alpha_{\mathrm{s}}$ and its running can be measured over a very large range in $\mathrm{Q}^{2}$ from hadronic inclusive and multi-jet rates in NC interactions. The combination [7] of the ZEUS and H1 published HERA I $\alpha_{\mathrm{s}}$ measurements yields $\alpha_{\mathrm{s}}\left(\mathrm{M}_{\mathrm{Z}}\right)=0.1198 \pm 0.0019$ (exp.) \pm 0.0026 (th.). The current best individual experimental measurement [8] has been obtained from normalized inclusive and multi-jet cross sections using the full $\mathrm{H} 1$ data sample. The resulting preliminary value $\alpha_{\mathrm{s}}\left(\mathrm{M}_{\mathrm{Z}}\right)=0.1182 \pm 0.0008$ (exp.) \pm 0.0018 (PDF) $+0.0041 /-0.0031$ (scale) quotes an experimental error of $0.7 \%$, which is much lower than the uncertainties associated to the current QCD calculations. For a full exploitation of the available experimental accuracy, improved QCD calculations at NNLO are mandatory.

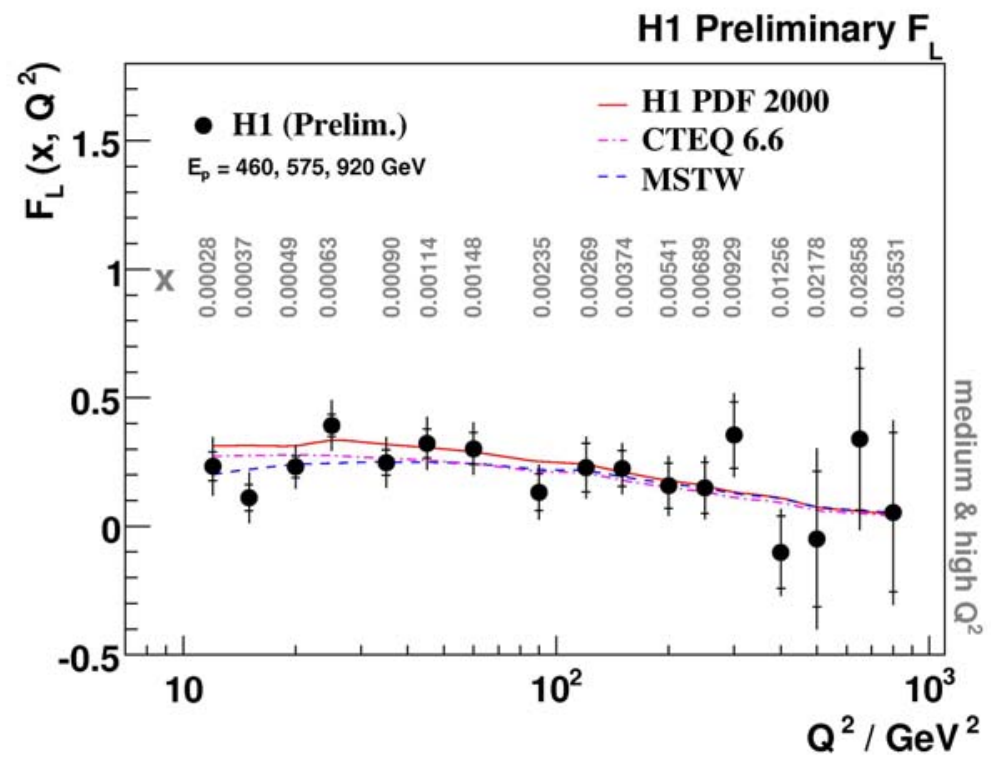

Figure 5: Longitudinal structure function $F_{L}$ averaged in $x$ as function of $Q^{2}$, as measured by $H 1$. 
The gluon content of the proton can be either derived indirectly from inclusive QCD fits, as discussed in the previous section, or probed directly from the measurement of $F_{L}$ and exclusive processes. The $F_{L}$ measurement is an experimental challenge since the highest sensitivity to $F_{L}$ is obtained with scattered electrons of very low energy, a domain where photoproduction background is high. Nevertheless the low proton energy runs taken from March to June 2007 were exploited in a record time by both experiments and first results were already presented in spring 2008 [9][10]. Figure 5 shows the latest H1 measurement [11] obtained in a large $\mathrm{Q}^{2}$ range. It agrees with theoretical predictions based on NLO QCD. The measurements are still expected to be extended to lower $\mathrm{Q}^{2}$ where the model predictions differ most.

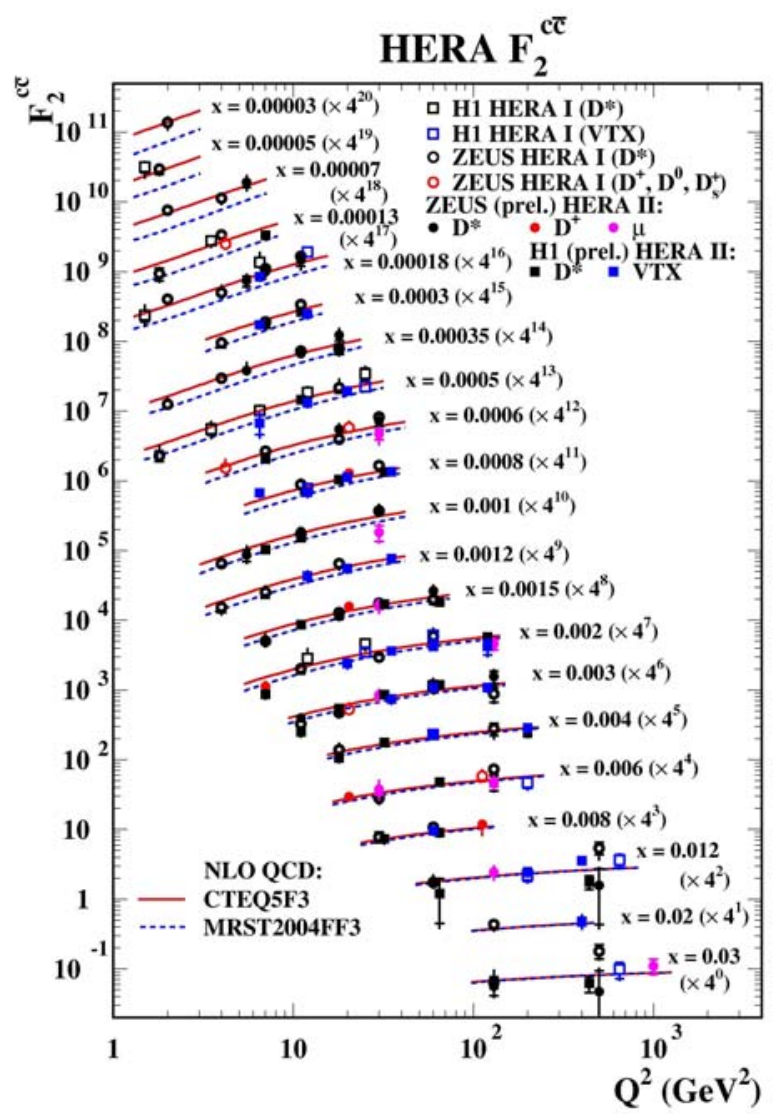

Figure 6: Charmed structure function $F_{2}^{c c}$ measured by $H 1$ and ZEUS with various charm tags, compared to NLO QCD predictions based on inclusive PDF fits.

Heavy flavour production is also directly sensitive to the gluon content since charm and beauty quarks in the proton are dynamically produced through photon-gluon fusion. At HERA heavy quarks can be tagged using various techniques involving $\mathrm{D}$ and $\mathrm{D}^{*}$ reconstruction, decay muon identification and displaced vertices tagged with silicon detectors. Figure 6 shows the current measurements of the charmed structure function $\mathrm{F}_{2}{ }^{\mathrm{cc}}$ obtained by $\mathrm{H} 1$ [12] and ZEUS [13]. In the HERA kinematic range charm production represents o $(25 \%)$ of the inclusive cross section. Similar results are available for beauty production [12][13], which accounts for a few $\%$ 
of deep inelastic interactions at HERA. Both charm and beauty dynamical generation are found to be adequately predicted by NLO DGLAP calculations based on the PDF fits to the inclusive cross-sections. This is a stringent consistency check of the overall QCD framework used to describe the data.

In summary both inclusive and exclusive tests of QCD dynamics support the validity of the DGLAP approximation in the bulk of the HERA phase space.

\section{Acknowledgements}

I am deeply indebted to my ZEUS and H1 Colleagues whose outstanding work made these results possible.

\section{References}

Preliminary results are available from the ZEUS and H1 web sites:

http://www-zeus.desy.de/public_results/publicsearch.html

http://www-h1.desy.de/h1/www/publications/H1preliminary.short list.html

[1] H1prelim-06-142 and ZEUS-prel-06-022.

[2] H1prelim-07-007 and ZEUS-prel-07-026.

[3] H1prelim-08-045 and ZEUS-prel-08-003.

[4] A.M. Cooper-Sarkar and E. Perez, HERA-LHC workshop, CERN, Geneva, 26-30 May 2008.

[5] E. Tassi, private communication.

[6] http://projects.hepforge.org/lhapdf/

[7] H1prelim-07-132 and ZEUS-prel-07-025.

[8] H1prelim-08-031.

[9] F.D. Aaron et al., H1 Collaboration, Phys. Lett. B665 139 [arxiv : 0805.2809 ].

[10] ZEUS-prel-08-001.

[11] H1 prelim-08-042.

[12] H1prelim-08-172, H1prelim-08-173 and H1 prelim-08-174.

[13] ZEUS-prel-07-008, ZEUS-prel-07-009 and ZEUS-prel-08-007. 